\title{
Relativistic loss cone dynamics: Infall and inspiral rates and branching ratios
}

\author{
Tal Alexander \\ Department of Particle Physics \& Astrophysics, Weizmann Institute of Science, \\ 234 Herzl St, Rehovot 76100, Israel \\ email: tal.alexander@weizmann.ac.il
}

\begin{abstract}
I describe recent advances in the formulation and modeling of relativistic stellar dynamics around a massive black hole, and the implications for the rates of infall (e.g. tidal disruption) and inspiral (e.g. gradual decay by gravitational wave emission) processes, and their branching ratios.
\end{abstract}

Keywords. stellar dynamics, black hole physics, Galaxy: center, galaxies: active, gravitational waves

\section{Introduction}

There are many pathways to the tidal disruption of a star by a massive black hole $(\mathrm{MBH})$ (Figure 1), which assume very different conditions (steady vs transient), dynamics (collisional vs non-collisional) or configurations (e.g. single vs binary MBH). Each further bifurcates into different outcomes (e.g. whether the interacting stars end up bound or unbound to the $\mathrm{MBH}$ ). Thus, the practical question for the planning and execution of observations: What is the theoretically predicted rate and properties of tidal disruption events (TDEs)? is too general for a useful answer. Theory takes initial and boundary conditions and projects a prediction. However, the initial and boundary conditions themselves are external to the logical chain of theoretical arguments, and unless introduced "by hand", must be supplied by observations. In that sense, all theoretical predictions are conditional.

Here I focus on a specific set of conditions, not because these are necessarily more realistic, but because the theory that describes them is more predictive and / or simpler and better developed. I will assume that the galactic nucleus is (1) in steady state, (2) that the dynamical processes responsible for supplying stars for TDEs are collisional, and (3) that only a single MBH exists in the nucleus.

The dynamics leading to such TDEs are a specific instance of the long-standing general stellar dynamical "loss-cone problem": How do stars in a galactic nucleus get to interact strongly with a $\mathrm{MBH}$, and / or fall into it, and at what rates? (Frank \& Rees 1976). The term loss-cone refers to the cone of velocity vectors that take a star from position $\boldsymbol{r}$ relative to the $\mathrm{MBH}$ to a periapse distance smaller than a critical value $r_{l c}$, where some strong interaction of interest, usually destructive, takes place (e.g. direct horizon crossing or stellar disruption). All stars that are initially on such orbits are quickly removed from the system. From that time on the loss rate is determined by the rate at which new stars are deflected into loss-cone orbits. Thus the loss-cone problem corresponds to the question: How do galactic nuclei randomize? (see Alexander 2017 for an extended review). 


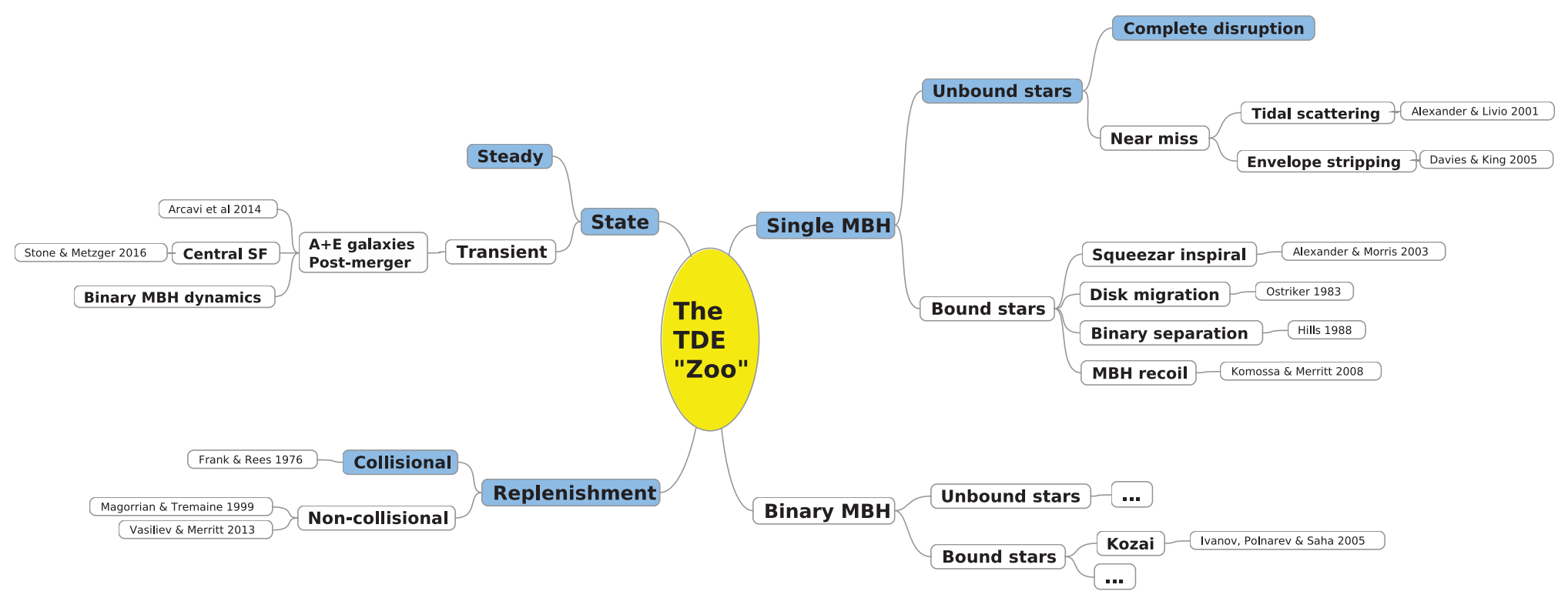

Figure 1. The theoretical TDE "zoo". A schematic of some of the many possibilities, permutations, configurations and dynamical processes that lead to TDEs and related phenomena. The options marked by a darker background are the prompt TDEs discussed here. 


\section{The classical loss-cone}

The classical analysis of the loss-cone problem (Lightman \& Shapiro 1977) focused on plunge processes, which require only a single approach to the $\mathrm{MBH}$, such as a TDE, and on scattering by incoherent 2-body interactions. Close to the $\mathrm{MBH}$, inside a critical radius $r_{d}$, the accumulated scatter per orbit is small relative the the angular opening of the loss-cone. Stars diffuse slowly into it, and are promptly lost. At any given time the number of stars on loss-cone orbits is negligible. This dynamical regime is called the empty or diffusive loss-cone, where the plunge rate is governed by the slow 2-body relaxation rate. Further out at $r>r_{d}$, the angular opening of the loss-cone is small enough that the scatter per orbit can cause a star to skip all across the loss-cone before it reaches periapse. This is the full loss-cone regime, where the plunge rate is set by the orbital period. A general analysis of the contributions to the total rate from both regimes (Syer \& Ulmer 1999) indicates that the total rate can be conservatively estimated (to within a factor of 2) by taking the smaller of $r_{d}$ and the radius of the $\mathrm{MBH}$ influence $r_{h}=G M_{\bullet} / \sigma^{2}$, where $\sigma$ is the velocity dispersion in the bulge of the host galaxy, $r_{e}=\min \left(r_{d}, r_{h}\right)$ and using the empty loss-cone plunge rate, $\Gamma_{p}=N_{\star}\left(<r_{e}\right) /\left[\log \left(J_{c}\left(r_{e}\right) / J_{l c}\right) T_{E}\left(r_{e}\right)\right]$, where $N_{\star}(<r)$ is the number of stars inside $r, J_{l c}=\sqrt{2 G M_{\bullet} r_{l c}}$ is the angular momentum of the loss-cone, $J_{c}$ is the maximal (circular) angular momentum, and $T_{E}$ the 2-body energy relaxation time.

A dissipative dynamical process can extract energy from the orbit (e.g. by gravitational wave (GW) emission (e.g. Amaro Seoane et al 2007), tidal heating (Alexander \& Morris 2003), or hydrodynamic interactions with a massive disk (Ostriker 1983)). The dissipation rate is usually a strong function of radius, and occurs mostly near periapse. A gradual inspiral into the MBH requires many repeated periapse passages. This can happen only on tight orbits with short periods, where orbital decay by dissipation is faster than scattering by the background stars, which can abort the inspiral by deflecting the star from its eccentric orbit to a nearly radial plunge orbit, or to a wider orbit, where dissipation is negligible. Figure (2a) shows two possible trajectories of stars in $(\log j, \log a)$ phasespace, where $j=J / J_{c}=\sqrt{1-e^{2}}$ is the normalized angular momentum, and $a$ the orbital semi-major axis (sma) in the Keplerian approximation. Plunges occur with high probability for stars with sma $a_{c}<a<a_{h}$ (typically $a_{c} \ll a_{h}=r_{h}$ ), while inspirals occur with high probability for stars with $a<a_{c}$. The inspiral to plunge event rate ratio reflects the ratio of the small number of stars in the real-space volume inside $a_{c}$, relative to the very large number, $N_{\star} \sim \mathcal{O}(Q)\left(Q=M_{\bullet} / M_{\star}\right)$, in the volume interior to $a_{h}$, $\Gamma_{i} / \Gamma_{p} \sim N_{\star}\left(<a_{c}\right) / N_{\star}\left(<a_{h}\right) \sim \mathcal{O}(0.01)$ (Alexander \& Hopman 2003).

\section{The relativistic loss-cone: GR precession and resonant relaxation}

Non-resonant (incoherent) two-body relaxation is inherent to any system of interacting discrete particles, and provides a minimal randomization rate. The point-like nature of gravitational interactions between stars boosts the relaxation timescale by the logarithmic Coulomb factor, which for a nearly Keplerian system around a MBH scales as $T_{N R} \propto\left[Q^{2} P / N_{\star}\right] / \log Q$, where $P$ is the orbital period. Non-resonant relaxation (NR) can change both the orbital energy and angular momentum.

There is however a restricted but much faster form of angular momentum relaxation, resonant relaxation (RR) (Rauch \& Tremaine 1996), which operates in nearly spherical potentials, like the one around a $\mathrm{MBH}$, where symmetry restricts orbital evolution (e.g. fixed ellipses in a Keplerian potential). A test star then moves in a static background potential generated by the $N_{\star}$ orbitally-averaged elliptical "mass wires", which conserve 


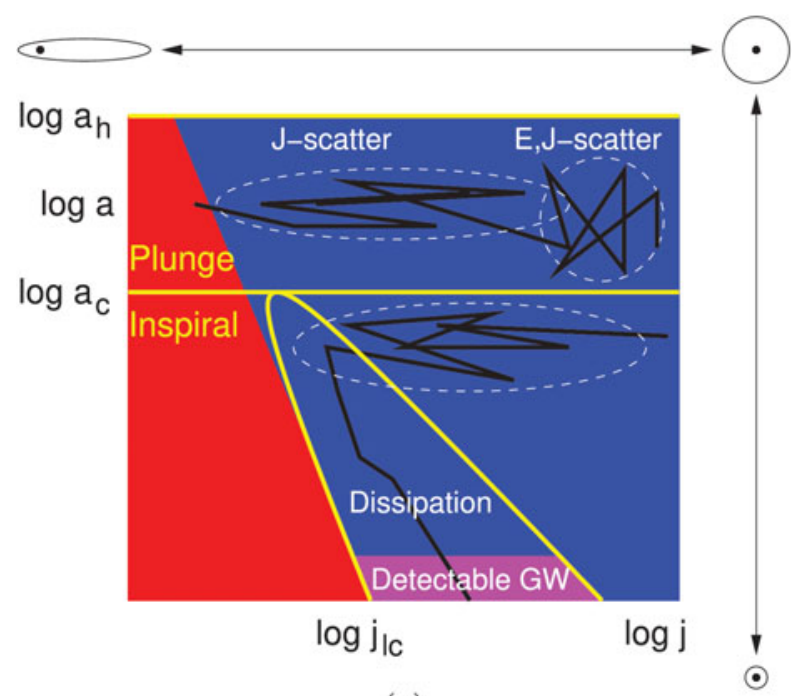

(a)

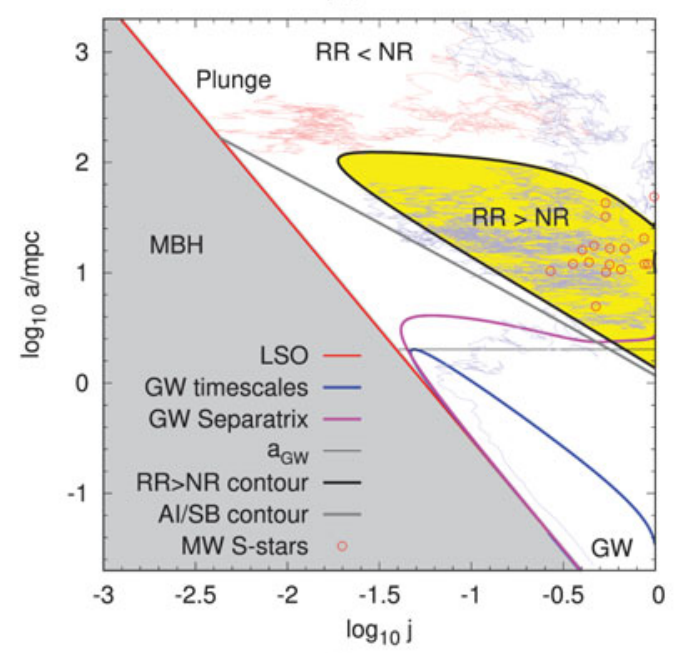

(b)

Figure 2. (a) A schematic of the "classical" loss-cone, where only 2-body incoherent relaxation is considered (see text). The time to change the angular momentum by order of itself, $T_{J}$, is related to the time to change the energy by order of itself, $T_{E}$, by $T_{J}=j^{2} T_{E}$. Stars on nearly circular orbits (upper right corner) are scattered equally in energy and angular momentum. However, once the star is scattered by chance to a somewhat eccentric orbit, scattering in angular momentum becomes much faster. When the orbit lies above the critical semi-major axis (sma) $a_{c}$ defined by the upper tip of the arc where GW dissipation is faster than the 2-body scatter, it will plunge with high probability directly into the MBH (the wedge of GR-unstable orbits on the left). However, when it lies below $a_{c}$, it will cross with high-probability through the GW dissipation region, where it is diverted to an inspiral orbit. (b) The phase space of the loss-cone as derived from the $\boldsymbol{\eta}$-formalism. The triangular region to the left is the region of unstable orbits that plunge into the MBH. The rounded region on the right is where RR dominates the dynamics. The diagonal line just below it is the SB/AI line, where RR is completely quenched by GR precession. The arc contour is the region where GW dissipation is faster than NR. The critical sma $a_{c}$ is at the tip of the arc. The curved line that overlaps with part of the arc is the exact separatrix between flow-lines that plunge and those that inspiral. Two stellar tracks are displayed as examples. The S-stars discovered around the Galactic MBH Sgr A* (circles) are observed to lie in the RR-dominated region (see text). (Bar-Or \& Alexander 2016). 
its energy, but exert a residual torque $\tau_{N} \sim \sqrt{N_{\star}} G M_{\star} / r$ that persists for a coherence time $T_{c}$ until the small deviations from the Keplerian potential, due to the distributed stellar mass ("mass precession"), to GR precession, or to the mutual resonant interactions between the background stars ("self-quenching") grow to $\mathcal{O}(1)$. The large coherent change in the test star's angular momentum over the coherence time, $\delta J$, then becomes the mean free path of a random walk in phase space on timescales $\mathrm{t} \gg T_{c}: \Delta J / J_{c} \sim\left(\delta J / J_{c}\right) \sqrt{t / T_{c}} \equiv$ $\sqrt{t / T_{R R}}$. The extended mass wires cannot approach each other as point masses do. However, the RR relaxation timescale is boosted by the long coherence time, $T_{R R} \propto$ $\left[Q^{2} P / N_{\star}\right] P / T_{c}$.

Close to a MBH where mass precession is slow, but far enough that GR is still negligible, the coherence time can be so long that $T_{R R} / T_{N R} \sim \log Q\left(P / T_{c}\right) \ll 1$, and RR dominates the dynamics. Since efficient RR torques can rapidly change $J \rightarrow J_{l c}$ and lead to a strong interaction with the $\mathrm{MBH}, \mathrm{RR}$ is of special relevance to the loss-cone problem. In fact, unchecked RR can drain all the stars from the inner parts of a stellar cusp by efficiently pushing them to plunge orbits (Bar-Or \& Alexander 2016). This would have dramatic effects: it would completely suppress GWs from extreme mass ratio inspirals (EMRIs), and would leave no stars or pulsars on relativistic orbits for testing strong gravity (e.g. Will 2008).

However, stars on eccentric orbits undergo substantial in-plane (Schwarzschild) GR precession. In their analysis of the implications of RR for EMRIs, Hopman \& Alexander (2006) made the "fortunate coincidence" conjecture: since the GR in-plane precession is a PN1 effect (i.e. an $\mathcal{O}\left(\beta^{2}\right)$ effect), whereas GW emission is a PN2.5 effect, the precession should kick in well before GW dissipation is important, and reverse the sign of the RR torques every half cycle, so that on average the effect of the RR torques should cancel out. In that way, by the time GW emission becomes substantial (it is unaffected by precession), the EMRI orbit will already be completely decoupled from the stellar background, and the EMRI's quasi-periodic GW emission should proceed unimpeded.

The first ever fully-self consistent post-Newtonian $N$-body simulation (Merritt et al.2011) revealed an unanticipated result. Not only did GR precession indeed quench RR well before the GW inspiral began, there appeared to be a barrier in phase space, denoted the "Schwarzschild Barrier" (SB), where the stars' RR-driven random walk in angular momentum toward $j \rightarrow 0$ was blocked (Figure $2 \mathrm{~b}$ ). Near the SB, the orbital parameters oscillate at the GR precession frequency for about a coherence time, and then the stars appear to "bounce" back from the barrier to higher $j$, instead of evolving to a plunge or EMRI orbit, as expected. Only occasionally does a star "jump" across the barrier and reach the $\mathrm{MBH}$. This behavior was explained in a preliminary analysis (Alexander 2010) as related to precession under the influence a residual dipole-like force. However, the SB phenomenon, its nature, implications and very existence remained controversial.

\section{The $\eta$-formalism for relativistic loss-cone dynamics}

The difficulty in incorporating $\mathrm{RR}$ in a formal description of dynamics around a $\mathrm{MBH}$ is due to the fact that this phenomenon lies in the interface between stochastic and deterministic processes. The standard description of collisional dynamics in terms of Markovian ("memory-less") diffusion is fundamentally inconsistent with RR's long coherence times.

The $\boldsymbol{\eta}$-formalism (Bar-Or \& Alexander 2014) bridges this gap by expanding the full orbitally averaged Hamiltonian of the test star and background, which expresses the interactions between mass ellipses, to 1st order in a generalized multipole expansion (the 
description of the ellipse field around the $\mathrm{MBH}$ require 3 angles, as opposed to the 2 of the standard multipole expansion of a scalar density field). The resulting expression for the background term is still too complex to solve analytically, but its symmetries can be analyzed, and reveal that to 1st order, the torques by the stellar background can be described as a vector $\boldsymbol{\eta}$ in angular momentum space. Since the change in the angular momentum of the test star is relatively small over $T_{c}$, this vector can be approximated as a function of time only. Since the residual torque by the background is due to a superposition of many ellipses, $\boldsymbol{\eta}(t)$ can be further approximated as a Gaussian random noise field. At this level of treatment the temporal behavior of the background noise has to be introduced in a non-self-consistent way (in contrast, the magnitude of the torque, $\tau_{N}$, is known to good approximation, see Sec. 3).

A key insight is that the nature of the resulting dynamics depends critically on the smoothness (differentiability) of the noise. The power spectrum of a smooth noise must have a maximal frequency above which the power decreases rapidly. For RR, this frequency is $\nu_{c} \sim \mathcal{O}\left(T_{c}^{-1}\right)$. When the precession frequency of the test star, $\nu_{G R}=\nu_{0} j^{-2}$ is higher than $\nu_{c}$, the star is decoupled from the noise: it's orbit is adiabatically invariant under the external perturbations. The SB is therefore the adiabatic invariance (AI) locus $j_{A I}(a)=\sqrt{\nu_{0} T_{c}(a) / 2 \pi}$ (Figure $2 \mathrm{~b}$ ). Beyond this line, RR is rapidly quenched by GR precession, and only NR affects the dynamics.

The first-order, orbit-averaged Hamiltonian can be integrated in time with a randomly generated realizations of the noise $\boldsymbol{\eta}(t)$, to produce realizations of stellar trajectories in phase space. This is useful for confirming that the various approximations that go into the $\boldsymbol{\eta}$ formalism reproduce the properties of the SB that are observed in the $N$ body simulations. However, the major practical step forward that is provided by the $\eta$-formalism is the derivation of effective diffusion coefficients that incorporate AI (BarOr \& Alexander 2014). These then allow to evolve in time the stellar distribution function by integrating the Fokker-Planck (diffusion) equation by the Monte Carlo method (BarOr \& Alexander 2016), which corresponds to modeling the realistic $N_{\star} \rightarrow \infty$ limit. By comparison, direct $N$-body (e.g. Merritt et al.2011) is currently limited to $\mathcal{O}(100)$ stars with an unrealistically small mass ratio $Q$. Another advantage of the Monte Carlo method is that it is simple to add NR, GW, tidal heating and other physical processes, and to switch on or off various physical mechanisms. The $\boldsymbol{\eta}$-formalism Monte Carlo results reproduce the low- $N_{\star} N$-body plunge and inspiral rates, and also the behavior observed near the SB/AI line (Bar-Or \& Alexander 2016).

\section{Results}

Figure (2b) shows the various critical lines in the phase-space of the loss-cone for a Milky Way-like Galaxy with $M_{\bullet}=4 \times 10^{6} M_{\odot}$ and $r_{h}=2 \mathrm{pc}$, overlayed with two Monte Carlo-generated stellar trajectories, one a plunge and the other a GW inspiral. $\mathrm{RR}$ dominates the dynamics only in a limited region that is away from the loss-lines (the last stable orbit or the GW line), and therefore RR does not affect much the loss-rates. Figure $(3 \mathrm{c})$ shows the dependence of the loss rates on $M_{\bullet}$, with and without RR. The difference is small - this confirms the fortunate coincidence conjecture (Sec. 3).

Over long timescales $\sim \mathcal{O}\left(T_{N R}\right)$, NR completely randomizes the distribution function in phase-space, and it approaches the expected maximal entropy configuration, irrespective of the short timescale behavior near the SB/AI line, since the steady state should not depend on the nature of the randomization process. This is shown in Figure (3a). A slight enhancement in the local plunge rate is seen where the RR-dominated region extends closest to the loss-line (Figure 3b). 


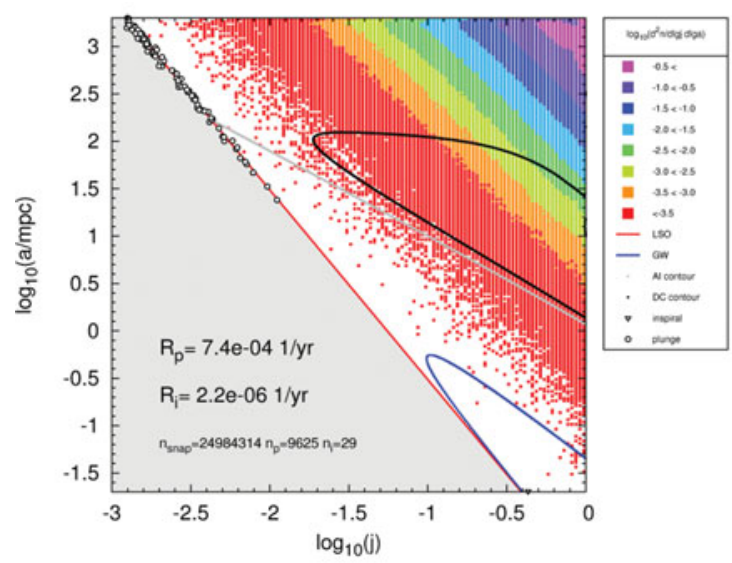

(a)

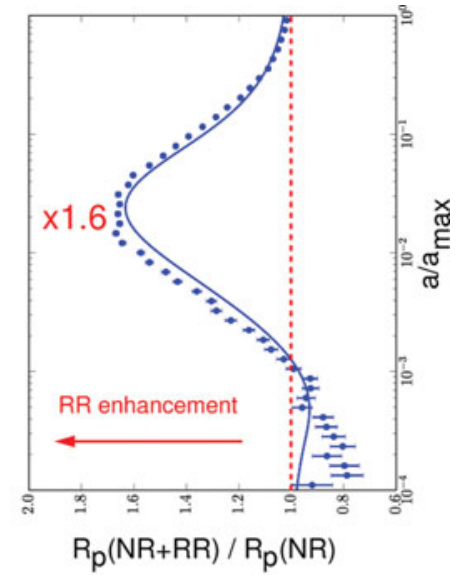

(b)

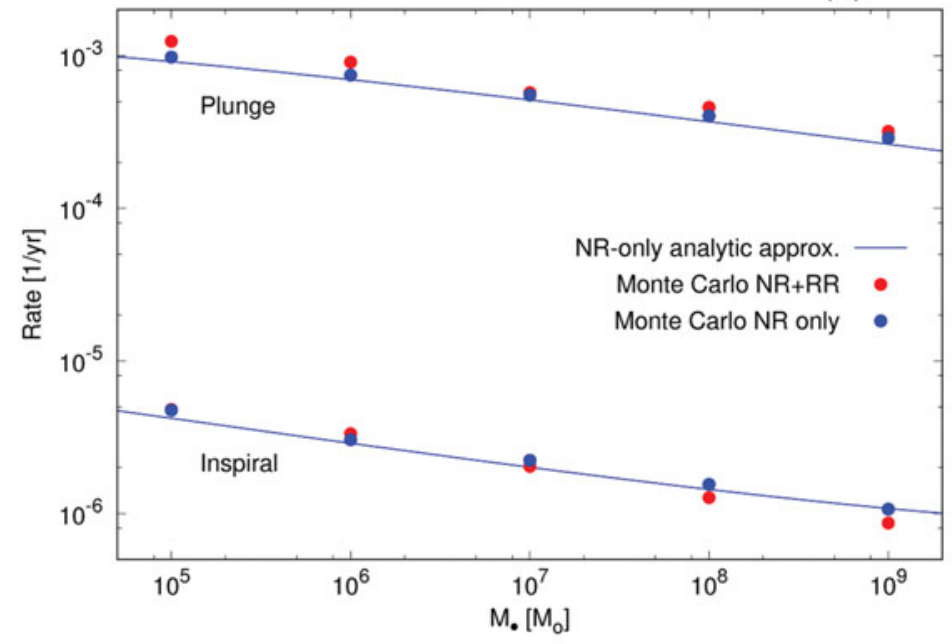

(c)

Figure 3. (a) An $\boldsymbol{\eta}$-formalism Monte Carlo simulation of a Milky Way-like galaxy, taking into account NR, RR, GR and mass precession, and assuming a smooth noise. The color scale shows the steady state stellar distribution function, the end-points of some of the stellar trajectories are shown as black circles, and the resulting steady-state loss rates are quoted on the left. (b) The small relative differential enhancement due to RR, as function of sma $a$, scaled to correspond to panel (a). (c) The steady state plunge and inspiral rates as function of MBH mass $M_{\bullet}$, with and without RR. (Adapted from Bar-Or \& Alexander 2016).

While RR does not affect much the steady state loss rates, it can have a strong effect on stars that happen to orbit in the RR-dominated region. The S-stars observed around the MBH of the Milky Way Sgr A* (main sequence B-stars) are such stars (Gillessen et al 2009). There are two leading scenarios for explaining their presence so close to the MBH. One is that they were tidally captured by the Hills mechanism (Hills 1988) (a 3-body interaction between the $\mathrm{MBH}$ and an incoming binary, where one star is captured on a tight eccentric orbit and the other is ejected as a hyper-velocity star), in which case their initial eccentricity was very high, and the other that they migrated from a stellar disk such as the one observed today around Sgr $A^{\star}$ (Levin 2007), in which case their initial eccentricity was low. Their presently observed eccentricity distribution is thermal 
(random), which requires post-formation / capture evolution of angular momentum. $\boldsymbol{\eta}$ formalism Monte Carlo experiments show (Sabsovich et al.2017) that for either scenarios to work, one must assume the existence of a dense "dark" cusp of remnants and lowluminosity stars that can exert the rapid RR torques on the S-stars. The simulations further indicate that the Hills mechanism is favored over the disk migration (see also Perets et al 2009). RR is likewise relevant for modeling the dynamics of relativistic stars and pulsars near Sgr A* for the purpose of testing strong gravity (e.g. Will 2008).

\section{References}

Alexander, T. 2010 in: P. Amaro-Seoane \& E. Porter (eds.), GW and EM signatures of MBH binaries and EMRIs, online proc. http://www.aei.mpg.de/ pau/conf_vid4/Alexander.pdf Alexander, T. 2017, ARAA in press.

Alexander, T. \& Livio, M. 2001, ApJ (Letters) 560, L143

Alexander, T. \& Morris, M. 2003, ApJ (Letters) 590, L25

Alexander, T. \& Hopman, C. 2003, ApJ (Letters) 590, L29

Amaro Seoane, P., et al. 2007, CQG 24, 113

Arcavi, I., et al. 2014, ApJ 793, 38

Bar-Or, B. \& Alexander, T. 2014, ApJ 820, 129

Bar-Or, B. \& Alexander, T. 2016, CQG 31, 244003

Davies, M. \& King, A. 2005, ApJ (Letters) 624, L25

Frank, J. \& Rees, M. J. 1976, MNRAS 176, 633

Gillessen, S., et al. 2009, ApJ 692, 362

Hills, J. G. 1988, Nature 331, 687

Hopman, C. \& Alexander, T. 2006, ApJ 645, 1152

Ivanov, P. B., Polnarev, A. G. \& Saha, P. 2005 MNRAS 358, 1361

Komossa, S. \& Merritt, D. 2008 ApJ (Letters) 683, L21

Levin, Y. 2007 MNRAS 374, 515

Lightman, A. P. \& Shapiro, S. L. 1977, ApJ 211, 244

Magorrian, J., et al. 1998 AJ 115, 2285

Merritt, D., Alexander, T., Mikkola, S., \& Will, C. 2011, PRD 84, 044024

Ostriker, J. P. 1983, ApJ 273, 99

Perets, H. B., et al. 2009, ApJ 702, 884

Rauch, K. P. \& Tremaine, S. 1996, New Astron. 1, 149

Sabsovich, D., Alexander, T. \& Bar-Or, B. 2017 in prep.

Stone, N. C. \& Metzger, B. D. 2016 MNRAS 455, 859

Syer, D. \& Ulmer, A. 1999, MNRAS 306, 35

Vasiliev, E. \& Merritt, D. 2013, ApJ 774, 87

Will, C. M. 2008, ApJ (Letters) 674, L25 